\title{
Factors Associated with Poor Treatment Outcome among Hospitalized COVID-19 Patients in South Central, Ethiopia
}

\author{
Abdene Weya Kaso $\mathbb{D}^{1},{ }^{1}$ Habtamu Endashaw Hareru ${ }^{D},{ }^{1}$ Taha Kaso ${ }^{(D)},{ }^{2}$ and Gebi Agero ${ }^{3}{ }^{3}$ \\ ${ }^{1}$ School of Public Health, Dilla University, Ethiopia \\ ${ }^{2}$ Department of Surgery, College of Health Science, Arsi University, Ethiopia \\ ${ }^{3}$ Department of Public Health, College of Health Science, Arsi University, Ethiopia
}

Correspondence should be addressed to Abdene Weya Kaso; abdannekaso@gmail.com

Received 10 November 2021; Revised 20 January 2022; Accepted 2 February 2022; Published 23 February 2022

Academic Editor: Mohd Saeed

Copyright (c) 2022 Abdene Weya Kaso et al. This is an open access article distributed under the Creative Commons Attribution License, which permits unrestricted use, distribution, and reproduction in any medium, provided the original work is properly cited.

\begin{abstract}
Background. Deaths due to COVID-19 are common among the elderly, especially among individuals with underlying illnesses. The pandemic of the COVID-19 impaired the mental, psychological, and physical well-being of people admitted to hospitals. Furthermore, in underdeveloped countries, scarcity of medical equipment was a challenge to manage cases in public health facilities. Thus, understanding the epidemiology and clinical outcomes of COVID-19 patients who are receiving treatment is critical for developing effective treatments and assessing service quality. Therefore, this study is aimed at assessing the treatment outcomes and associated factors among patients affected by the COVID-19 virus. Method. We used an institutionalbased retrospective cross-sectional analysis of 398 patients discharged in South Central, Ethiopia, between June 1, 2020, and July 5, 2021. Data were extracted using the data abstraction format. Data were entered, coded, and analyzed using the STATA 16 software. Bivariate and multivariate logistic regression analysis was used to assess the factors associated with poor treatment outcomes. A 95\% confidence interval with adjusted odds ratio (AOR) and $p$ value less than 0.05 were considered statistically significant. Result. In our study, the proportion of poor treatment outcomes was 61 (15.3\%). Chronic pulmonary disease $(\mathrm{AOR}=5.62$; 95\% CI: 2.49-12.70), asthma $(\mathrm{AOR}=2.8 ; 95 \% \mathrm{CI}: 1.17-6.67)$, chronic kidney disease $(\mathrm{AOR}=4.81 ; 95 \% \mathrm{CI}: 1.27-$ 18.22), diabetic mellitus ( $\mathrm{AOR}=2.27 ; 95 \% \mathrm{CI}: 1.02-5.09$ ), $\mathrm{HIV}$ positive ( $\mathrm{AOR}=10.44 ; 95 \% \mathrm{CI}$ : 3.0-36.35), worsening conditions ( $\mathrm{AOR}=3.73,95 \% \mathrm{CI}: 1.17-11.95)$, and age 55 and above years (AOR $=4.35,95 \% \mathrm{CI}$ : $1.30-14.60$ ) were statistically associated with poor treatment outcomes.Conclusion. We found a significant number of patients had favourable treatment. Moreover, aging, having complicated situations at admission, and chronic illnesses such as COPD, CKD, asthma, diabetic mellitus, and HIV/AIDS participants were significantly associated with poor treatment outcomes. Therefore, critical follow-up and management of patients with underlying diseases and worsening health conditions during admission is required.
\end{abstract}

\section{Background}

Coronaviruses (CoVs) are RNA viruses that are singlestranded, encapsulated, and belong to the Coronaviridae family $[1,2]$. They are classified as beta coronaviruses, gamma coronaviruses, and delta coronaviruses based on differences in protein sequences. The human coronaviruses (HCoV) HCoV-OC43, HCoV-229E, HCoV-NL63, and HCoVHKU1 are among the less pathogenic $\mathrm{HCoV}$ that cause a mild common cold or diarrhea [3]. Currently, three coronavirus infections have been identified, all of which are highly pathogenic and cause mild to severe respiratory tract disorders [4]. Among these, the recently discovered severe acute respiratory syndrome coronavirus (SARS-CoV), which was connected to a pneumonia outbreak in 2003 and had an 11 percent fatality rate, was linked to a pneumonia outbreak. The most prevalent clinical signs and symptoms of SARS$\mathrm{CoV}$ were viral pneumonia, fever, chills, myalgia, and a nonproductive cough, with sore throats being less common. The virus has a two-to-seven-day incubation period [5]. The Middle East respiratory syndrome coronavirus (MERS$\mathrm{CoV}$ ) was first found in Saudi Arabia in 2012 and has a 
fatality rate of $35 \%$. The virus has a two to fourteen-day incubation period, and symptoms include fever, shortness of breath, diarrhea, vomiting, cough, sore throat, and stomach discomfort, with a substantial number of critically ill patients requiring ICU treatment $[3,5]$.

The novel coronavirus (SARS-CoV-2) is a highly transmissible and dangerous virus that first appeared in Wuhan, China, in December $[4,6]$. The virus is a beta coronavirus that causes human sickness and is transmitted through coughing and sneezing droplets. At room temperature, it can survive for up to six days on contaminated surfaces and objects. Infected patients experienced fever, dry cough, and shortness of breath as a result of the infection $[7,8]$. The World Health Organization (WHO) declared the epidemic a global pandemic after it spread to other countries [9]. As of July 5, 2021, there had been 183,198,019 confirmed cases and 3,971,687 deaths worldwide [10]. On March 13, 2020, Ethiopia reported the first confirmed case of COVID-19. Until July 5, 2021, Ethiopia reported 276,323 confirmed cases and 4,327 deaths due to the COVID-19 outbreak $[11,12]$. In hospitalized patients, the case fatality rate (CFR) was estimated to be roughly $5 \%$, with an overall mortality rate of 0.25 percent among confirmed cases. COVID19 infection fatality rates increased with age, and patients with underlying diseases had a higher death rate [13]. Approximately, $20 \%$ of patients admitted to the hospital had severe symptoms and were admitted to the ICU [4, 14]. The COVID-19 pandemic impaired people's emotional, psychological, and physical wellbeing while they were in hospitals [15-19]. The recent finding revealed that individual perceptions regarding a virus had a significant impact on the outcome of treatment. Moreover, medical and supportive care equipment shortages in low and middleincome countries (LMIC) hampered the treatment of illnesses in public health facilities [14]. Understanding the epidemiological and clinical results of COVID-19 patients undergoing therapy, in contrast, is critical for determining the efficacy of therapies and assessing service quality [20]. COVID-19 research has primarily concentrated on prevention measures, epidemiological inquiry, diagnosis, and therapy to date. To our knowledge, no studies have assessed the clinical outcomes and risk factors of COVID-19 patients admitted to hospitals in low-income countries, particularly Ethiopia. As a result, the goal of this study was to determine the treatment outcomes and associated characteristics among COVID-19 patients in Ethiopia's South Central region.

\section{Method and Materials}

2.1. Study Design, Population, and Setting. From September 1 to 15,2021 , we conducted an institutional-based retrospective cross-sectional study among hospitalized COVID-19 patients in the Arsi Zone of Ethiopia. There are 28 woredas and two town administrations in the Arsi zone. COVID-19 patients were treated in this zone at the Bokoji hospital treatment center, which is 56 kilometres from the zonal town of Assela. The study covered all COVID-19 patients who were treated between June 1, 2020, and July 5, 2021. Patients with incomplete medical records were excluded from the COVID-19.

2.2. Sample Size and Sampling Technique. We employed a single population proportion with a $95 \%$ confidence level, $Z / 2=1.96,5 \%$ margin of error, design effect $=1.5$, and the proportion of patients who had poor treatment outcomes (41\%) [21]. We employed a correction formula because the number of patients discharged was fewer than 10,000, and the final sample for this study was 398 COVID-19 patient records. The treatment center was chosen using a multistage sampling technique, and the medical records of COVID-19 patients were assessed using a systematic random sample technique.

2.3. Study Variables and Operational Definition. In accordance with WHO and Ethiopian COVID-19 treatment and discharge protocol, we defined the final treatment outcome of patients under treatment as cured, transfer out, discharged with consent, or death $[22,23]$. When patients were cured or discharged with a physician order for home-based treatment, the treatment outcome of the COVID-19 patient was considered favourable. Furthermore, if the COVID-19 patients died in the treatment center or were transferred out for further medical treatment to nearby treatment centers, the treatment outcome was rated poor. In this study, cured patients were defined as COVID-19 patients who had two consecutive negative polymerase chain reaction (PCR) results after 14 days. Moreover, patients who had positive PCR findings after 14 days in the hospital and were discharged for home-based treatment after clinical improvement were considered discharged with consent. Patients who died from any cause in the treatment facility after being hospitalized with COVID-19 were defined as death, whereas patients who were transferred out were defined as patients who were referred to nearby treatment facilities for an additional investigation related to any cause or COVID-19. The sociodemographic factors, comorbidity conditions, clinical characteristics, and status at admission were independent variables.

2.4. Data Collection Procedure and Quality Management. We retrieved data from patients' medical records using an abstraction format prepared for the study. Thus, data on patients' sociodemographic, admission status, intranasal oxygen use, place of care, length of stay, comorbidity status, and outcome were collected from COVID-19 medical records. After one day of training, two nurses extracted data, and the collected data were reviewed for consistency and completeness daily. The overall activities of data extraction were supervised by the principal investigator.

2.5. Data Analysis. The extracted data were entered, coded, cleaned, and analyzed using STATA version 16 . We calculated the mean and standard deviations (SD) for continuous data and used frequency and percentage to describe categorical variables. Furthermore, to determine the factors associated with the outcome variable, bivariate logistic regression analyses with a 95 percent confidence level and crude odds ratio (COR) were performed, and variables with a $p$ value 
less than 0.25 in the bivariate analysis were considered for multivariate logistic regression analysis. Finally, 95 percent confidence intervals with adjusted odds ratios of less than 0.05 were declared to have a significant association with the outcome variable.

2.6. Ethics Approval and Consent to Participate. We obtained ethical approval for this study from Arsi University. Consent to participate was waived since the study was conducted through a review of medical records. Individual patients were not subject to any hurt, and the data was not used for other purposes.

\section{Result}

3.1. Patient's Sociodemographic Characteristics. One hundred fifty-one $(37.9 \%)$ patients were from urban areas, and $237(59.5 \%)$ of participants were males. The majority (25.4\%) of patients were above 54 years old, with a mean age of patients 39 (SD: 19.4 years) (Table 1).

3.2. Patients' Clinical Characteristics. Among 374 patients with clinical signs and symptoms, 82 (20.6\%) were mild, $104(26.1 \%)$ were moderate, and $170(42.7 \%)$ were severe, whereas $18(4.5 \%)$ were critical at admission. A total of 21.9 percent of the 188 patients with worsening symptoms had chronic pulmonary disease (COPD), 19.6 percent had diabetic mellitus, and 17.8 percent had asthma (Figure 1). More than half of the patients $(59.3 \%)$ had comorbidities, with diabetic mellitus (28\%) being the most frequent chronic condition, followed by COPD (17.1\%) and hypertension $(16.1 \%)$. Forty $(10 \%)$ of the patients were treated in the ICU and 250 (66.8) received intranasal oxygen care. The average length of stay was 13 (SD: 5.7) days (Table 2).

3.3. Patient Treatment Outcome. Among 398 COVID-19 patients, $337(84.7 \%)$ had favourable treatment outcomes, while $(2.8 \%)$ transferred out and $(12.5 \%)$ died in the treatment center (Figure 2).

3.4. Factors Associated with Poor Treatment Outcome of COVID-19 Patients. Variables like age, sex, residency, comorbidities, and admission status were statistically associated with poor treatment outcomes in bivariate logistic regression. When compared to their counterparts, the odds of poor treatment outcome among COVID-19 patients were significantly higher among COPD $(\mathrm{AOR}=5.62 ; 95 \% \mathrm{CI}$ : 2.49-12.70), asthmatic ( $\mathrm{AOR}=2.8 ; 95 \% \mathrm{CI}: 1.17-6.67)$, CKD $(\mathrm{AOR}=4.81 ; 95 \% \mathrm{CI}: 1.27-18.22)$, diabetic mellitus $(\mathrm{AOR}=2.27 ; 95 \%$ CI: 1.02-5.09), and HIV positive $(\mathrm{AOR}=10.44 ; 95 \% \mathrm{CI}: 3.0-36.35)$. Furthermore, people aged 55 and above years old $(\mathrm{AOR}=4.35,95 \% \mathrm{CI}$ : $1.30-$ 14.60) were nearly four times more likely to have poor treatment outcomes compared to 0-24 years. On the other hand, patients who were in severe or critical conditions during admission were 3.73 times more likely to have poor treatment outcomes $(\mathrm{AOR}=3.73$, 95\% CI: 1.17-11.95) (Table 3).
TABLE 1: Sociodemographic characteristics of COVID-19 patients admitted to the treatment center in Arsi zone, 2021.

\begin{tabular}{lcc}
\hline Variable & Categories & Frequency (\%) \\
\hline \multirow{2}{*}{ Sex } & Male & $237(59.5)$ \\
& Female & $161(40.5)$ \\
\hline \multirow{2}{*}{ Age category } & $0-24$ year & $98(24.6)$ \\
& $25-34$ year & $91(22.9)$ \\
& $35-44$ year & $56(14.1)$ \\
Mean age & $45-54$ & $52(13.1)$ \\
\hline \multirow{2}{*}{ Residence } & Above 55 years & $101(25.4)$ \\
& & \\
\hline
\end{tabular}

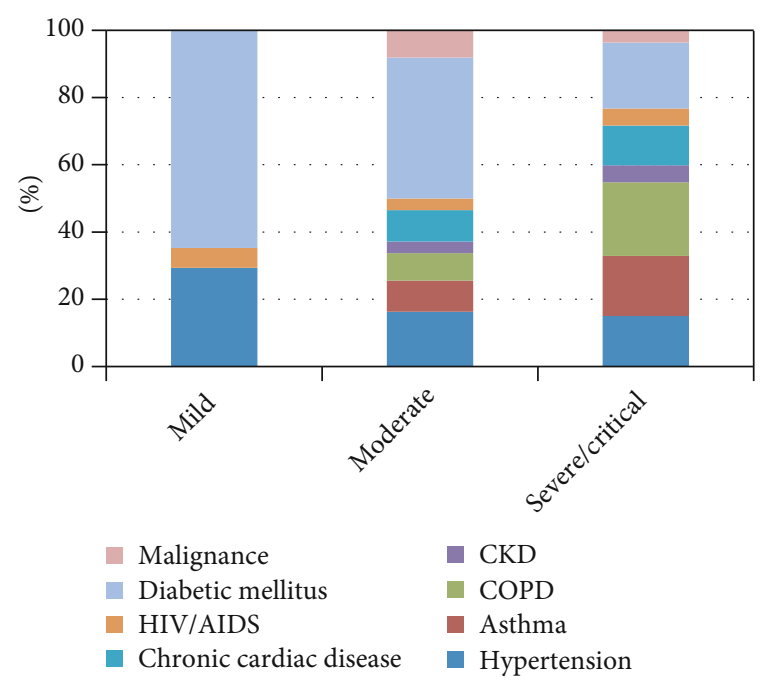

Figure 1: General health status of the patients at admission compared by types of chronic illnesses.

\section{Discussion}

The COVID-19 pandemic is a worldwide public health issue that has resulted in increased anxiety, death, and deterioration of health in people with comorbidities and the elderly $[24,25]$. In this study, we found that $15.3 \%$ of COVID-19 patients had poor treatment outcomes, which is similar to the report from Wuhan Pulmonary Hospital (11.73\%) [26] and higher than Ethiopian findings (1.9\%) [27]. On the contrary, this study's poor treatment outcome was lower than that of Belgium (29.9\%) [28] and China (28.27\%) [29]. These disparities could be the result of differences in care quality, sample size, age, comorbidities, study time, and settings. We discovered that the likelihood of a poor treatment outcome rises with age. Older patients were more likely than younger patients to have poor treatment results, according to studies from China [13, 29-32], Kurdistan [33], Saudi Arabia [34], and England [35]. This could be related to the body's immune defense system deteriorating with age, and older persons were more prone to severe disease and poor 
TABLe 2: Clinical characteristics of patients admitted with COVID-19 to the treatment center in Arsi zone, 2021.

\begin{tabular}{|c|c|c|}
\hline Clinical characteristics & Categories & Frequency (\%) \\
\hline \multirow{2}{*}{ Clinical manifestation } & Yes & $374(94)$ \\
\hline & No & $24(6.0)$ \\
\hline \multirow{2}{*}{ Co-morbidity } & Yes & $236(59.3)$ \\
\hline & No & $162(40.7)$ \\
\hline \multirow{5}{*}{ Status } & Asymptomatic & $24(6.0)$ \\
\hline & Mild & $82(20.6)$ \\
\hline & Moderate & $104(26.1)$ \\
\hline & Severe & $170(42.7)$ \\
\hline & Critical & $18(4.5)$ \\
\hline \multirow{7}{*}{ Type of comorbidity } & Hypertension & $52(16.1)$ \\
\hline & Chronic cardiac disease & $34(10.6)$ \\
\hline & COPD & $55(17.1)$ \\
\hline & Asthma & $47(14.6)$ \\
\hline & Chronic kidney disease (CKD) & $14(4.3)$ \\
\hline & Diabetic mellitus & $90(28.0)$ \\
\hline & Human immune virus (HIV) & $15(4.7)$ \\
\hline \multirow{2}{*}{ Intranasal oxygen use } & Yes & $250(66.8)$ \\
\hline & No & $124(33.2)$ \\
\hline \multirow{2}{*}{ Place of care } & Ward & $358(90.0)$ \\
\hline & $\mathrm{ICU}$ & $40(10.0)$ \\
\hline \multirow{3}{*}{ Length of stay } & Less than 15 days & $263(66.1)$ \\
\hline & 15 and above days & $135(33.9)$ \\
\hline & Mean(SD) & $13(5.7)$ \\
\hline
\end{tabular}

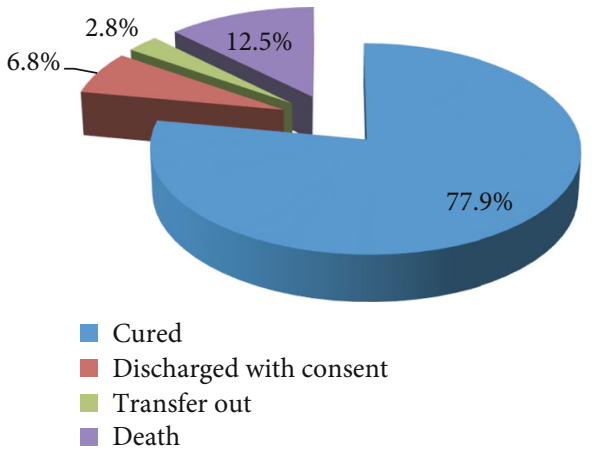

Figure 2: Treatment outcome of Patients with COVID-19 at the treatment center in Arsi zone, 2021.

treatment outcomes from COVID-19 infection because they were more likely to have many chronic conditions that hampered their health [36].

Our study found that diabetic mellitus patients had higher odds of COVID-19 poor treatment compared to those without disease. Previous studies found that the presence of diabetes increased mortality in patients with COVID19, which was consistent with our finding [30, 33, 37-39]. Individuals with COPD, and asthma, had also considerably worse treatment outcomes than those who did not have the disease $(p<0.05)$. This is consistent with a study from Italy [40], and China [26, 30, 41], Germany [42], and Saudi Arabia [34], which found that patients with heart disease, asthma, and COPD had a poor COVID-19 prognosis. In addition, HIV positive and CKD COVID-19 patients had higher odds of poor treatment outcome compared to their counterpart. These could be due to patients with these comorbidities are more prone to acquire a more severe health condition and disease development, increasing their susceptibility to bad outcomes [43].

In this study, when compared to their counterparts, patients with severe/critical conditions during admission were more likely to have poor treatment outcomes. The study from China [44] and India [45] also reported the same trends among patients with worsening health conditions during admission. This can be explained by the fact that persons who were in those situations may have a chronic illness that worsens their health and leads to poor treatment outcomes. Even though we evaluated patients' treatment outcomes in a health center that served patients from 28 different districts, our study had significant limitations. First, because the data for the study was gathered from secondary sources, incomplete patient information posed a significant barrier. Second, due to the retrospective nature of the study design, all factors that were not available on registration were not included in the analysis. Moreover, the cross- 
TABLE 3: Bivariate and multivariate logistic regression analysis of factors associated with the treatment outcome of COVID-19 patients, 2021.

\begin{tabular}{|c|c|c|c|c|}
\hline \multirow{2}{*}{ Categories } & \multicolumn{2}{|c|}{ Treatment outcome } & \multirow{2}{*}{ COR $(95 \%$ CI $)$} & \multirow{2}{*}{ AOR (95\% CI) } \\
\hline & Favourable (\%) & Poor (\%) & & \\
\hline \multicolumn{5}{|l|}{ Age } \\
\hline $0-24$ year & $91(92.9)$ & $7(7.1)$ & 1 & 1 \\
\hline 25-34year & $85(93.4)$ & $6(6.6)$ & $2.12(0.61,7.36)$ & $0.83(0.18,3.94)$ \\
\hline $35-44$ years & $49(87.5)$ & $7(12.5)$ & $3.95(1.15,13.64)$ & $1.06(0.24,4.61)$ \\
\hline 45-54 years & $43(84.3)$ & $9(15.7)$ & $4.16(1.20,14.36)$ & $2.64(0.62,11.16)$ \\
\hline 55 and above years & $69(68.3)$ & $32(31.7)$ & $4.77(1.62,14.07)$ & $4.35(1.30,14.60)^{*}$ \\
\hline \multicolumn{5}{|l|}{ Sex } \\
\hline Female & $137(85.1)$ & $24(14.9)$ & $0.95(0.54,1.66)$ & $1.14(0.56,2.34)$ \\
\hline Male & $200(84.4)$ & $37(15.6)$ & 1 & 1 \\
\hline \multicolumn{5}{|l|}{ Residence } \\
\hline Rural & $132(87.4)$ & $19(12.6)$ & 1 & 1 \\
\hline Urban & $205(83.0)$ & $42(17.0)$ & $1.42(0.79,2.55)$ & $1.26(0.62,2.57)$ \\
\hline \multicolumn{5}{|l|}{ Hypertension } \\
\hline Yes & $40(76.9)$ & $12(23.1)$ & $1.82(0.89,3.71)$ & $1.58(0.68,3.69)$ \\
\hline No & $297(85.8)$ & $49(14.2)$ & 1 & 1 \\
\hline \multicolumn{5}{|l|}{ Chronic cardiac disease } \\
\hline Yes & $26(76.47)$ & $8(23.53)$ & $1.81(0.78,4.20)$ & $2.46(0.85,7.09)$ \\
\hline No & $311(85.4)$ & $53(14.6)$ & 1 & 1 \\
\hline \multicolumn{5}{|l|}{ COPD } \\
\hline Yes & $32(58.2)$ & $23(41.8)$ & $5.78(3.06,10.87)$ & $5.62(2.49,12.70)^{*}$ \\
\hline No & $305(88.9)$ & $38(11.1)$ & 1 & 1 \\
\hline \multicolumn{5}{|l|}{ Asthma } \\
\hline Yes & $32(68.1)$ & $15(31.9)$ & $3.11(1.56,6.18)$ & $2.80(1.17,6.67)^{*}$ \\
\hline No & $305(86.9)$ & $46(13.1)$ & 1 & 1 \\
\hline \multicolumn{5}{|l|}{ CKD } \\
\hline Yes & $7(50.0)$ & $7(50.0)$ & $6.11(2.06,18.11)$ & $4.81(1.27,18.22)^{*}$ \\
\hline No & $330(85.9)$ & $54(14.1)$ & 1 & 1 \\
\hline \multicolumn{5}{|l|}{ Diabetic mellitus } \\
\hline Yes & $73(81.1)$ & $17(18.9)$ & $1.27(0.68,2.36)$ & $2.27(1.02,5.09)^{*}$ \\
\hline No & $264(85.7)$ & $44(14.3)$ & 1 & 1 \\
\hline \multicolumn{5}{|l|}{ Malignance } \\
\hline Yes & $10(66.67)$ & $5(33.33)$ & $2.92(0.96,8.86)$ & $2.29(0.45,11.78)$ \\
\hline No & $327(68.9)$ & $56(31.1)$ & 1 & 1 \\
\hline \multicolumn{5}{|l|}{ HIV/AIDS } \\
\hline Positive & $6(40.0)$ & $9(60)$ & $9.55(3.26,27.94)$ & $10.44(3.0,36.35)^{*}$ \\
\hline Negative & $331(86.4)$ & $52(13.6)$ & 1 & 1 \\
\hline \multicolumn{5}{|l|}{ Admission status } \\
\hline No symptom/mild & $102(96.2)$ & $4(3.8)$ & 1 & 1 \\
\hline Moderate & $94(90.4)$ & $10(9.6)$ & $2.17(0.72,6.58)$ & $2.14(0.58,7.93)$ \\
\hline Severe/critical & $141(75)$ & $47(25)$ & $6.66(2.56,17.34)$ & $3.73(1.17,11.95)^{*}$ \\
\hline
\end{tabular}

Note: ${ }^{*} p$ value $<0.05$ 
sectional nature of the study design also does not indicate the cause and effect relationship between the factors.

\section{Conclusion}

In this study, we found that a significant number of patients had favourable treatment. Moreover, having complicated situations at admission, and chronic illnesses such as COPD, CKD, asthma, diabetic mellitus, and HIV/AIDS, participants were significantly associated with poor treatment outcomes. We found that respondents aged 55 and above years old were also associated with poor treatment outcomes. Therefore, critical follow-up and management of patients with underlying diseases and worsening health conditions during admission is required.

\section{Abbreviations}

AIDS: Acquired immune deficiency syndrome

AOR: Adjusted odds ratio

CI: Confidence interval

CKD: Chronic kidney disease

COPD: Chronic pulmonary disease

CoVs: Coronaviruses

COR: Crude odds ratio

HIV: Human immune virus

$\mathrm{HCoV}$ : Human coronavirus

LMIC: Low and medium-income countries

ICU: Intensive care unit

MERS: Middle East respiratory syndrome

PCR: Polymerase chain reaction

OR: Odds ratio

SARS: Severe acute respiratory syndrome

SD: $\quad$ Standard deviation

WHO: World Health Organization.

\section{Data Availability}

The datasets supporting the conclusions of this article are included in the supporting information.

\section{Conflicts of Interest}

The authors declare that they have no competing interests.

\section{Acknowledgments}

Our deepest gratitude goes to Bokoji hospital staff for their continuous support during data collection. Our appreciation also goes to Mr. Mengesha Moges and Mr. Angasa Demissie for their unreserved inputs during data collection.

\section{References}

[1] I. Salahshoori, N. Mobaraki-Asl, A. Seyfaee et al., "Overview of COVID-19 disease: virology, epidemiology, prevention diagnosis, treatment, and vaccines," Biologics, vol. 1, no. 1, pp. 240, 2021.
[2] F. Li, "Structure, function, and evolution of coronavirus spike proteins," Annual Review of Virology, vol. 3, no. 1, pp. 237261, 2016.

[3] Z.-W. Ye, S. Yuan, K.-S. Yuen, S.-Y. Fung, C.-P. Chan, and D.Y. Jin, "Zoonotic origins of human coronaviruses," International Journal of Biological Sciences, vol. 16, no. 10, pp. 16861697, 2020.

[4] W. J. Wiersinga, A. Rhodes, A. C. Cheng, S. J. Peacock, and H. C. Prescott, "Pathophysiology, transmission, diagnosis, and treatment of coronavirus disease 2019 (COVID-19)," Journal of the American Medical Association, vol. 324, no. 8, pp. 782-793, 2020.

[5] N. N. C. House and S. Palissery, "Corona viruses: a review on SARS, MERS and COVID-19," Microbiology Insights, vol. 14, p. $8,2021$.

[6] M. A. Shereen, S. Khan, A. Kazmi, N. Bashir, and R. Siddique, "COVID-19 infection: emergence, transmission, and characteristics of human coronaviruses," Journal of Advanced Research, vol. 24, pp. 91-98, 2020.

[7] A. Hafeez, S. Ahmad, S. A. Siddqui, M. Ahmad, and S. Mishra, "A review of COVID-19 (coronavirus Disease-2019) diagnosis, Treatments and Prevention," Eurasian Journal of Medicine and Oncology, vol. 4, no. 2, pp. 116-125, 2020.

[8] Solution, Clarivate Analytics, Disease Briefing: Coronaviruses, Clarivate Analytics, Philadelphia, PA, 2020.

[9] S. S. Unhale, Q. B. Ansar, S. Sanap et al., "A review on corona virus (covid-19)," World Journal of Pharmaceutical and Life Sciences, vol. 6, no. 4, pp. 109-115, 2020.

[10] "Weekly Operational Update on COVID-19," 2021, https:// www.who.int/publications/m/item/weekly-operationalupdate-on-covid-19.

[11] FMOH, Covid-19 daily situational analysis, Addis Ababa, Ethiopia, 2021.

[12] "Covid-19 daily situational analysis update," 2020, May 2020.

[13] Q. Shi, X. Zhang, F. Jiang et al., "Clinical characteristics and risk factors for mortality of COVID-19 patients with diabetes in Wuhan, China: a two-center, retrospective study," Diabetes Care, vol. 43, no. 7, pp. 1382-1391, 2020.

[14] E. J. Emanuel, G. Persad, R. Upshur et al., "Fair Allocation of Scarce Medical Resources in the Time of Covid-19," The New England Journal of Medicine, vol. 382, no. 21, pp. 2049-2055, 2020.

[15] W. Zhang, K. Wang, L. Yin et al., "Mental health and psychosocial problems of medical health workers during the COVID19 epidemic in China," Psychotherapy and Psychosomatics, vol. 89, pp. 242-250, 2020.

[16] R. Chou, T. Dana, D. I. Buckley, S. Selph, R. Fu, and A. M. Totten, "Update alert: epidemiology of and risk factors for coronavirus infection in health care workers," Annals of Internal Medicine, vol. 173, no. 2, pp. W46-W47, 2020.

[17] N. Suryavanshi, A. Kadam, G. Dhumal et al., "Mental health and quality of life among healthcare professionals during the COVID-19 pandemic in India," Brain and Behavior, vol. 10, no. 11, article e01837, 2020.

[18] M. Kurnik-Łucka, P. Pasieka, A. Górecka-Mazur, E. RząsaDuran, J. Pękala, and K. Gil, Health-related quality of life of pharmacists in the pre-COVID-19 era, 2020.

[19] J. Stojanov, M. Malobabic, G. Stanojevic, M. Stevic, V. Milosevic, and A. Stojanov, "Quality of sleep and healthrelated quality of life among health care professionals treating 
patients with coronavirus disease-19," International Journal of Social Psychiatry, vol. 67, no. 2, pp. 175-181, 2021.

[20] E. E. Alzoubi, R. Hariri, and N. J. Attard, "Oral health related quality of life impact in dentistry," Journal of Dental Health Oral Disorders \& Therapy, vol. 6, no. 6, pp. 35-42, 2017.

[21] M. Arab-Zozani, F. Hashemi, H. Safari, M. Yousefi, and H. Ameri, "H A. Health-related quality of life and its associated factors in COVID-19 patients," Osong Public Health and Research Perspectives, vol. 11, no. 5, pp. 296-302, 2020.

[22] WHO, Clinical management of COVID-19: interim guidance, 27 May 2020, World Health Organization, 2020.

[23] FMOH, National Guideline for Integration of Covid-19 and Essential Healthcare Services, Addis Ababa, Ethiopia, 2021.

[24] F. D. Algahtani, S. U. Hassan, B. Alsaif, and R. Zrieq, “Assessment of the quality of life during COVID-19 pandemic: a cross-sectional survey from the Kingdom of Saudi Arabia," International Journal of Environmental Research and Public Health, vol. 18, p. 847, 2021.

[25] F. Greco, V. M. Altieri, F. Esperto, V. Mirone, and R. M. Scarpa, "Impact of COVID-19 pandemic on health-related quality of life in uro-oncologic patients: what should we wait for?," Clinical Genitourinary Cancer, vol. 19, no. 2, pp. e63e68, 2021.

[26] L. L. R. Du RH, C. Q. Yang, W. Wang et al., "Predictors of mortality for patients with COVID-19 pneumonia caused by SARSCoV-2: a prospective cohort study," The European Respiratory Journal, vol. 55, no. 5, p. 2000524, 2020.

[27] B. M. Gebremariam, K. L. Shienka, B. A. Kebede, and M. G. Abiche, "Epidemiological characteristics and treatment outcomes of hospitalized patients with COVID-19 in Ethiopia," The Pan African Medical Journal, vol. 37, no. 7, 2020.

[28] J. Meester, D. Bacquer, M. Naesens, B. Meijers, M. M. Couttenye, and A. S. Vriese, "Incidence, characteristics, and outcome of COVID-19 in adults on kidney replacement therapy: a regionwide registry study," Journal of the American Society of Nephrology, vol. 32, no. 2, pp. 385-396, 2021.

[29] F. Zhou, T. Yu, R. Du et al., "Clinical course and risk factors for mortality of adult inpatients with COVID-19 in Wuhan, China: a retrospective cohort study," Lancet, vol. 395, no. 10229, pp. 1054-1062, 2020.

[30] C. Yu, Q. Lei, W. Li et al., "Clinical characteristics, associated factors, and predicting COVID-19 mortality risk: a retrospective study in Wuhan, China," American Journal of Preventive Medicine, vol. 59, no. 2, pp. 168-175, 2020.

[31] L. L. R. Du RH, C. Q. Yang, W. Wang et al., "Predictors of mortality for patients with COVID-19 pneumonia caused by SARS-CoV-2: a prospective cohort study," The European Respiratory Journal, vol. 55, no. 5, p. 2000524, 2020.

[32] R. Chen, W. Liang, M. Jiang et al., "Risk factors of fatal outcome in hospitalized subjects with coronavirus disease 2019 from a Nationwide analysis in China," Chest, vol. 158, no. 1, pp. 97-105, 2020.

[33] E. Zandkarimi, G. Moradi, and B. Mohsenpour, "The prognostic factors affecting the survival of Kurdistan Province COVID-19 patients: a cross-sectional study from February to May 2020," International Journal of Health Policy and Management, pp. 1-6, 2020.

[34] A. Khan, S. Althunayyan, Y. Alsofayan et al., "Risk factors associated with worse outcomes in COVID-19: a retrospective study in Saudi Arabia," Eastern Mediterranean Health Journal, vol. 26, no. 11, pp. 1371-1380, 2020.
[35] E. Williamson, A. Walker, K. Bhaskaran et al., "Factors associated with COVID-19-related death using OpenSAFELY," Nature, vol. 584, no. 7821, pp. 430-436, 2020.

[36] T. W. Leulseged, D. G. Alemahu, I. S. Hassen et al., "Factors associated with development of symptomatic disease in Ethiopian COVID-19 patients: a case-control study," BMC Infectious Diseases, vol. 21, no. 1, pp. 1-7, 2021.

[37] B. L. Altonen, T. M. Arreglado, O. Leroux, M. Murray-Ramcharan, and R. Engdahl, "Characteristics, comorbidities and survival analysis of young adults hospitalized with COVID19 in New York City," PLoS One, vol. 15, no. 12, 2020.

[38] M. C. Chang, Y. K. Park, B. O. Kim, and D. Park, "Risk factors for disease progression in COVID-19 patients," BMC Infectious Diseases, vol. 20, no. 1, pp. 1-6, 2020.

[39] J. Song, D. W. Park, J. H. Cha et al., "Clinical course and risk factors of fatal adverse outcomes in COVID-19 patients in Korea: a nationwide retrospective cohort study," Scientific Reports, vol. 11, no. 1, pp. 1-11, 2021.

[40] G. Boari, G. Chiarini, S. Bonetti et al., "Prognostic factors and predictors of outcome in patients with COVID-19 and related pneumonia: a retrospective cohort study," Bioscience Reports, vol. 40 , no. $12,2020$.

[41] L. Wang, W. He, X. Yu et al., "Coronavirus disease 2019 in elderly patients: characteristics and prognostic factors based on 4-week follow-up," Journal of Infection, vol. 80, no. 6, pp. 639-645, 2020.

[42] I. Nachtigall, P. Lenga, K. Jóźwiak et al., "Clinical course and factors associated with outcomes among 1904 patients hospitalized with COVID-19 in Germany: an observational study," Clinical Microbiology and Infection, vol. 26, no. 12, pp. 16631669, 2020.

[43] D. L. Ramatillah, S. H. Gan, S. A. S. Sulaiman et al., "Evaluation of treatment outcome for pneumonia among pre-vaccinated COVID-19 patients with/without comorbidity in a public hospital in Bengkulu, Indonesia," Vaccine, vol. 9, no. 12, p. 1411, 2021.

[44] L. Hu, S. Chen, Y. Fu et al., "Risk factors associated with clinical outcomes in 323 COVID-19 patients in Wuhan, China," Clinical Infectious Diseases, vol. 71, no. 16, 2020.

[45] V. Mishra, A. D. Burma, S. K. Das, M. B. Parivallal, S. Amudhan, and G. N. Rao, "COVID-19-hospitalized patients in Karnataka: survival and stay characteristics," Indian Journal of Public Health, vol. 64, Supplement, pp. S221-S224, 2020. 